\title{
Author Correction: Room-temperature ductile inorganic semiconductor
}

Xun Shi, Hongyi Chen, Feng Hao, Ruiheng Liu, Tuo Wang, Pengfei Qiu, Ulrich Burkhardt, Yuri Grin and Lidong Chen

Correction to: Nature Materials https://doi.org/10.1038/s41563-018-0047-z, published online 9 April 2018.

In the version of this Article originally published, the $x$-axis numbers of Fig. $3 \mathrm{~d}$ were incorrect; the range should have been 0 to 12 instead of 1 to 13 . This has now been corrected. 